\title{
Transverse Thermal Conductivity of REBCO Coated Conductors
}

\author{
M. Bonura, C. Senatore
}

\begin{abstract}
REBCO coated conductors (CCs) have the potential to widen considerably the application areas of superconductivity. Quench protection of REBCO-based devices represents one of the major obstacles to this expansion. Thermal conductivity data are mandatory ingredients for quench simulation studies. In particular, the transverse thermal conductivity $\left(\kappa_{T}\right)$ of the CC plays a key role in case of quenches in superconducting magnets. In the present work, we report $\kappa_{T}$ of thermally stabilized REBCO CCs produced by different manufacturers. We have measured $\kappa_{T}$ on single CCs rather than on stacks of soldered tapes, this excludes spurious contributions in the measurements. We have found that the presence of the stabilizer at the tape edges in $\mathrm{Cu}$ electroplated CCs increases the overall $\kappa_{T}$. In the absence of this effect, the overall thermal resistance is dominated by the substrate.
\end{abstract}

Index Terms-Thermal conductivity, thermal stability, coated conductors, magnet technology.

\section{INTRODUCTION}

$\mathrm{H}$ IGH-TEMPERATURE SUPERCONDUCTORS (HTS) have been the subject of extensive researches in recent years. The acquired expertise associated to major advances in the fabrication techniques has made REBCO coated conductors (CCs) promising candidates for many applications, such as solenoid and accelerator magnets, fault current limiters, and current cables [1-4].

Thermal properties of REBCO CCs play an important role in the design of HTS-based devices, since they determine the thermal stability. In particular, the thermal conductivity $(\kappa)$ of the $\mathrm{CC}$ is a mandatory parameter for modeling heat transfer $[5,6]$. In superconducting windings, both the longitudinal $\left(\kappa_{L}\right)$ and the transverse $\left(\kappa_{T}\right)$ thermal conductivities, which respectively describe thermal conduction along and across the tape, are needed [5,7]. $\kappa_{L}$ and $\kappa_{T}$ assume very different values in REBCO CCs, being $\kappa_{L}>\kappa_{T}$ [8]. In thermally stabilized CCs, longitudinal heat transfer mainly occurs through the stabilizer (usually $\mathrm{Cu}$ ). Since thermal conduction in a pure metal is ruled by the electronic contribution, it follows that $\kappa_{L}$ depends on the purity of the stabilizer as well as on the

The authors acknowledge the financial support from the Swiss National Science Foundation (Grant N. PP00P2-14467 and 51NF40-144613). Research also supported by FP7 EuCARD-2 http://eucard2.web.cern.ch. EuCARD-2 is co-funded by the partners and the European Commission under Capacities 7th Framework Programme, Grant Agreement 312453.

The authors are with the Department of Applied Physics (GAP) and the Department of Condensed Matter Physics (DPMC), University of Geneva, Quai Ernest Ansermet 24, CH-1211 Geneva, Switzerland (e-mail: marco.bonura@unige.ch; carmine.senatore@unige.ch). magnetic field $[9,10]$. Transverse heat exchange is also influenced by non- $\mathrm{Cu}$ layers and in particular by the substrate, which considerably lowers the overall thermal conductivity [11].

The normal zone propagation velocity (NZPV) is a significant parameter for the protection of superconducting devices. Low NZPV values represent one of the main obstacles to the use of REBCO CCs [12,13]. The detection of a quench and the consequent activation of the protection system depends on NZPV. Furthermore, high NZPV values allow limiting the maximum temperature attained during a quench. In case of adiabatic superconducting magnets, the transverse component of NZPV, $v_{T}$, plays a key role in the quench process [14]. The experimental determination of $v_{T}$ is much more complex than that of the longitudinal NZPV, $v_{L}$. Since $v_{L}$ and $v_{T}$ are correlated parameters, the latter can be evaluated from $v_{L}$ and the ratio of the transverse and longitudinal thermal conductivities: $v_{T}=v_{L} \sqrt{\kappa_{T} / \kappa_{L}}$ [5].

Very few experimental works on the thermal conductivity of REBCO tapes have been reported in the literature $[4,8]$. Furthermore, transverse thermal properties have never been examined on single CCs but only on stacks of tapes $[8,15]$. In a recent work, we have investigated the longitudinal thermal conductivity of industrial REBCO CCs [9]. In this manuscript, we report the transverse thermal conductivity of REBCO tapes produced by different manufacturers. These data can be of particular interest for the magnet design community.

\section{SAMPLES}

We have investigated REBCO CCs from five different manufacturers, namely American Superconductor (AMSC), Bruker HTS (BHTS), Fujikura, SuNAM, and SuperPower. The tape layout as well as the constituent materials may differ from one manufacturer to the others. Table I summarizes some of the CCs' properties interesting for this study.

AMSC and BHTS use Ni5at.\%W (NiW) and stainless steel for the substrate, respectively. The other manufacturers employ Hastelloy. Each manufacturer has his own recipe for the buffer layers (details in Table I). The thickness of the REBCO layers is about 1-2 $\mu \mathrm{m}$ for all the CCs [2]. A silver layer of about 1-2 $\mu \mathrm{m}$ is deposited over the REBCO layer for protection against the moisture from the environment. In the tape manufactured by SuperPower, a second Ag layer is also present below the substrate. All the CCs investigated in this work have been stabilized with $\mathrm{Cu}$ either by electroplating (BHTS, SuNAM, and Superpower) or by lamination (AMSC and Fujikura). 
TABLE I

DETAILS ON THE LAYOUT OF INVESTIGATED COATED CONDUCTORS

\begin{tabular}{|c|c|c|c|}
\hline Manufacturer & Substrate & Buffer Layers & Stabilization \\
\hline AMSC & Ni5at.\%W & $\mathrm{Y}_{2} \mathrm{O}_{3} / \mathrm{YSZ} / \mathrm{CeO}_{2}$ & Cu laminated \\
\hline BHTS & Stainless Steel & $\mathrm{YSZ} / \mathrm{CeO}_{2}$ & $\mathrm{Cu}$ electroplated \\
\hline FUJIKURA & Hastelloy & $\begin{array}{l}\mathrm{Al}_{2} \mathrm{O}_{3} / \mathrm{Y}_{2} \mathrm{O}_{3} / \\
\mathrm{MgO} / \mathrm{CeO}_{2}\end{array}$ & $\mathrm{Cu}$ laminated \\
\hline SUNAM & Hastelloy & $\begin{array}{l}\mathrm{Al}_{2} \mathrm{O}_{3} / \mathrm{Y}_{2} \mathrm{O}_{3} / \\
\mathrm{MgO} / \mathrm{LaMnO}_{3}\end{array}$ & $\mathrm{Cu}$ electroplated \\
\hline SUPERPOWER & Hastelloy & $\begin{array}{l}\mathrm{Al}_{2} \mathrm{O}_{3} / \mathrm{Y}_{2} \mathrm{O}_{3} / \\
\mathrm{MgO} / \mathrm{LaMnO}_{3}\end{array}$ & $\mathrm{Cu}$ electroplated \\
\hline
\end{tabular}

The CC from Fujikura has $\mathrm{Cu}$ just on one face of the tape. AMSC uses two $\mathrm{Cu}$ strips, one for each tape face, soldered together at the $\mathrm{CC}$ edges. Electroplated $\mathrm{CCs}$ have $\mathrm{Cu}$ all around the tape, edges included.

In Table II, we have reported other CCs' properties of interest for this study, namely the overall thickness and the thickness of substrate and stabilizer, and the $\mathrm{Cu}$ residual resistivity ratio $R R R \equiv R(273 \mathrm{~K}) / R(4.2 \mathrm{~K})$ obtained by measuring the electrical resistivity on $\mathrm{Cu}$ samples extracted from the CCs [9]. We have determined the layers' dimensions by optical microscopy.

TABLE II

PROPERTIES OF INVESTIGATED COATED CONDUCTORS

\begin{tabular}{lllll}
\hline \hline \multicolumn{1}{c}{ Manufacturer } & $\begin{array}{c}\text { Overall } \\
\text { Thickness }\end{array}$ & $\begin{array}{c}\text { Substrate } \\
\text { Thickness }\end{array}$ & $\begin{array}{c}\text { Copper } \\
\text { Thickness }\end{array}$ & $\begin{array}{c}\text { Copper } \\
R R R\end{array}$ \\
\hline AMSC & $200 \mu \mathrm{m}$ & $75 \mu \mathrm{m}$ & $100 \mu \mathrm{m}$ & 19 \\
BHTS & $150 \mu \mathrm{m}$ & $100 \mu \mathrm{m}$ & $30 \mu \mathrm{m}$ & 17 \\
FUJIKURA & $160 \mu \mathrm{m}$ & $75 \mu \mathrm{m}$ & $75 \mu \mathrm{m}$ & 59 \\
SUNAM & $110 \mu \mathrm{m}$ & $60 \mu \mathrm{m}$ & $36 \mu \mathrm{m}$ & 61 \\
SUPERPOWER & $100 \mu \mathrm{m}$ & $50 \mu \mathrm{m}$ & $40 \mu \mathrm{m}$ & 42 \\
\hline
\end{tabular}

\section{EXPERIMENT}

\section{A. Experimental Method}

We have investigated the thermal conductivity using a laboratory-made setup specifically designed for measurements on superconducting wires and tapes. $\kappa_{T}$ is defined as

$$
\kappa_{T}=\frac{Q}{\Delta T} \frac{t}{A},
$$

where $Q$ is the heat supplied to one face of the tape, $\Delta T$ the temperature gradient between the two faces, $t$ the thickness of the $\mathrm{CC}$ and $A$ the surface of the sample. For our setup, $A$ is typically $\approx 20 \mathrm{~mm}^{2}$.

Transverse thermal conductivity measurements in single CCs require a high sensitivity of the experimental apparatus. The reduced thickness of the tape can make $\Delta T$ so small that its experimental determination can be challenging [8]. We have taken specific precautions for the samples' preparation. In Fig. 1, we report a schematic drawing of the sample holder. The CC is sandwiched between two copper leads glued on the sample's faces by GE varnish. This ensures good thermal contact over the entire tape surface. The leads are in high- $R R R$ $\mathrm{Cu}$ and the temperature gradient along the leads is negligible with respect to $\Delta T$. A hole is present on each $\mathrm{Cu}$ lead in correspondence of the center of the sample. This allows gluing a Cernox bare chip, used as thermometer, directly on the tape

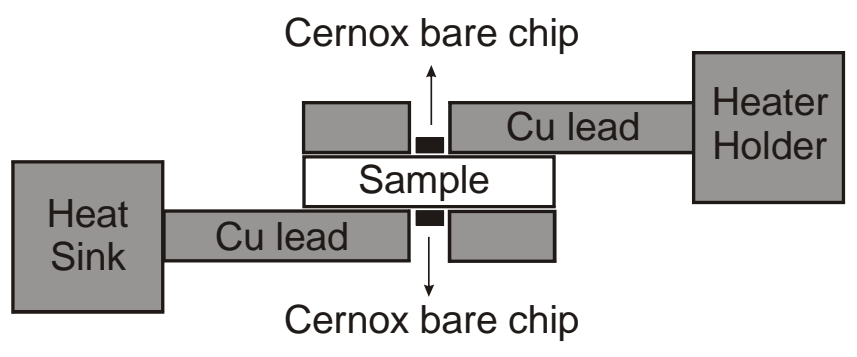

Fig. 1. Sketch of the sample holder.

face. Two calibrated Cernox thermometers are also present in the setup, one on the heat sink and one on the heater holder. During the measurement, the heat-sink temperature is kept constant. When current is supplied to the heater, energy is generated by Joule effect and a temperature gradient is established between the two sample faces. $\Delta T$ is measured once the steady-state heat flow has been reached. Typically $\Delta T$ is of the order of $10 \mathrm{mK}$.

The high values for the derivative of the resistance vs temperature curve of the Cernox bare chips $(d R / d T>1 \mathrm{k} \Omega / \mathrm{K}$ for $T<10 \mathrm{~K}$ ), permit an easy detection of temperature variations as small as $0.1 \mathrm{mK}$. Bare chips are mounted on the sample and their calibration is performed when the sample is at the thermal equilibrium with the bath. This removes possible errors associated to the thermal contact between the chips and the sample surface due to the GE varnish. The value of $d R / d T$ becomes smaller as temperature increases, and this leads to a growing uncertainty in the measurement. For this reason, we have investigated $\kappa_{T}$ up to $T \approx 25-30 \mathrm{~K}$. In the case of the CCs from BHTS and SuperPower, smaller $\kappa_{T}$ values (larger $\Delta T$ ) have allowed us to extend the investigation up to $T \approx 35 \mathrm{~K}$.

\section{B. Measurement Error}

The measurement uncertainty is mostly due to the evaluation of the ratio $t / A$, the associated maximum error being about $30 \%$. The error on $Q$ is smaller than the $0.05 \%$, since we have taken specific precautions for limiting convective, conductive and radiating heat losses [16]. Errors associated to $\Delta T$ increase with the temperature. This is a consequence of the characteristic $R(T)$ curve of Cernox bare chips. The maximum error associated to $\Delta T$ in the range of temperatures investigated is $15 \%$. For $T<10 \mathrm{~K}$ this percentage is smaller than $5 \%$.

\section{RESUltS AND DisCUSSION}

In Fig. 2, we report the temperature dependence of the transverse thermal conductivity for all the investigated samples. CCs from different manufacturers show different $\kappa_{T}$ values because of the different layouts and materials used. For all the tapes, we have observed an increase of $\kappa_{T}$ with the temperature.

Transverse thermal conduction in layered CCs can be analyzed with a formalism analogous to the case of electrical resistances connected in series. In Fig. 3, we show a simplified illustration of two different categories of $\mathrm{CCs}$ with the 
associated thermal/electrical circuit. $R_{O-L}$ indicates the thermal resistance associated to all the materials other than $\mathrm{Cu}$ and substrate. The configuration shown in Fig. 3 (a) is particularly appropriate to the $\mathrm{CC}$ from Fujikura, where $\mathrm{Cu}$ has been laminated only on one side of the tape. The overall thermal resistance is the sum of the resistances associated to each layer:

$$
R_{\mathrm{tot}}=\sum_{i} \frac{1}{\kappa_{i}} \frac{t_{i}}{A}+R_{\text {Int }},
$$

where $\kappa_{i}$ is the thermal conductivity associated to the $i^{\text {th }}$ layer, whose thickness is $t_{i}$. In (2) we have also included the term $R_{\text {Int }}$ associated to the interface thermal resistance due to a non-perfect connection between different layers.

In Fig. 3 (b) we show the typical cross-section of a $\mathrm{Cu}-$ electroplated CC. The stabilizer is deposited all around the tape. Thus, the contribution to the overall thermal transport from the $\mathrm{Cu}$ at the tape edges has to be considered, too. Transverse thermal transport in the $\mathrm{Cu}$-laminated $\mathrm{CC}$ from AMSC can also be schematized following the circuit in Fig. 3 (b). In this $\mathrm{CC}$, the upper and lower $\mathrm{Cu}$ strips are jointed together at the tape edges by a metal solder.

In the case of the $\mathrm{CC}$ from Fujikura, the absence of $\mathrm{Cu}$ on the tape edges allows us to use (2) for analyzing the experimental results of Fig. 2. $\kappa$ values of the $\mathrm{Cu}$ present in the $\mathrm{CC}$ can be deduced from longitudinal thermal conductivity measurements, since $\kappa_{L} \approx \kappa_{C u} s_{C u}$, where $s_{C u}$ is the cross-section fraction occupied by the stabilizer [9]. The temperature dependence of $\kappa_{L}$ for the CCs investigated in this work have been reported in Ref. [9]. It is worth analyzing the thermal behavior of the $\mathrm{CC}$ at temperatures close to values corresponding to the current sharing between superconductor and metal layers. In the case of REBCO CCs operating at $T=4.2 \mathrm{~K}$, this typically occurs between $25 \mathrm{~K}$ and $35 \mathrm{~K}[9,17]$. We deduce from Ref. [9] that the value of $\kappa_{C u}$ at $T=25 \mathrm{~K}$ is $\approx 1740 \mathrm{WK}^{-1} \mathrm{~m}^{-1}$. Lu et al. have reported on thermal conductivity of Hastelloy in a wide range of temperatures [18]. At $T=25 \mathrm{~K}$ they have found $\kappa_{\text {Hastelloy }} \approx 5 \mathrm{WK}^{-1} \mathrm{~m}^{-1}$. In the $\mathrm{CC}$ from Fujikura, $\mathrm{Cu}$ and Hastelloy layers have the same thickness $(t=75 \mu \mathrm{m})$. From (2), it follows that $R_{\text {Hastelloy }} / R_{C u}=$

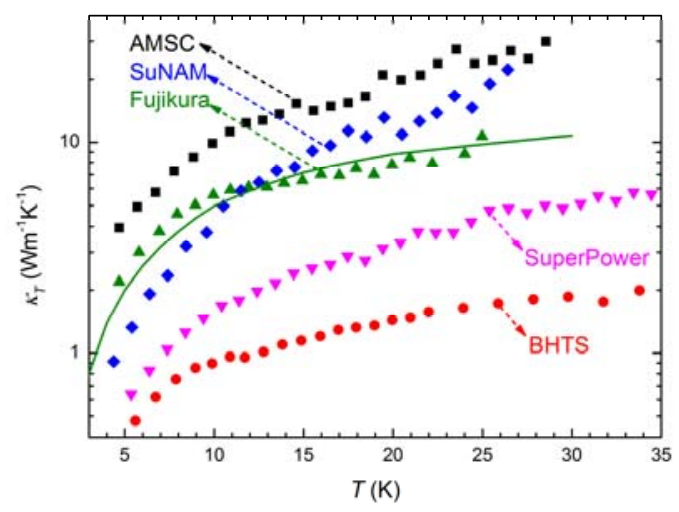

Fig. 2. Temperature dependence of the transverse thermal conductivity of REBCO coated conductors from different manufacturers. The continuous line indicates the expected curve for the tape from Fujikura.
$\kappa_{C u} / \kappa_{\text {Hastelloy }}$ and we can straightforwardly deduce that the contribution from the $\mathrm{Cu}$ layer to the overall thermal resistance can be neglected, being more than 2 orders of magnitude smaller than the one associated to the substrate. Using $\kappa(T)$ values of Hastelloy from Ref. [18] and expression reported in (2), we can calculate the expected value of $\kappa_{T}(T)$ for the Fujikura tape, in the hypothesis $R_{I n t}=R_{O-L}=0$. The calculated curve is reported in Fig. 2 as a continuous line. We have found a very good agreement between experimental and calculated data in the entire range of temperatures investigated. This indicates that for the $\mathrm{CC}$ from Fujikura, transverse thermal resistivity is dominated by the substrate contribution.

The same analysis cannot be performed for the CCs from the other manufacturers. This is a consequence of the contribution to the transverse thermal transport from the $\mathrm{Cu}$ (solder in the case of AMSC) on the tape edges. An illustrative example comes from the analysis of the results for the SuNAM tape. We can schematize thermal transport as in the circuit of Fig. 3 (b). Considering $\kappa$ values for the Hastelloy reported in Ref. [18] and using the geometrical specifications of the investigated sample ( $t_{\text {Hastelloy }} \approx 60 \mu \mathrm{m}, A \approx 20 \mathrm{~mm}^{2}$ ), we find: $R_{\text {sub }} \approx 0.6 \mathrm{~W}^{-1} \mathrm{~K}$ at $T=25 \mathrm{~K}$. We can also estimate the thermal resistance associated to the $\mathrm{Cu}$ present on the edges of the tape, $R^{\prime}{ }_{\mathrm{Cu}}$. From the results reported in Ref. [9] for the CC from SuNAM, we deduce that $\kappa_{C u} \approx 1910 \mathrm{WK}^{-1} \mathrm{~m}^{-1}$ at $T=25 \mathrm{~K}$. We have measured by optical microscopy the average thickness of the lateral $\mathrm{Cu}$ and it resulted to be $\approx 40 \mu \mathrm{m}(2 \mathrm{x}$ $20 \mu \mathrm{m})$. The length of the sample is $\approx 5 \mathrm{~mm}$, and thus we infer that $R^{\prime}{ }^{\prime} \approx 0.2 \mathrm{~W}^{-1} \mathrm{~K}$. This would indicate that at $T=25 \mathrm{~K}$ about $2 / 3$ of the overall thermal current passes through the $\mathrm{Cu}$ present at the edges of the $\mathrm{CC}$. It is noteworthy that the thermal conductivity of materials composing the $\mathrm{CC}$, including $\mathrm{Cu}$ and Hastelloy, are temperature dependent $[9,10,11,18]$. This could change the fractional amount of heat
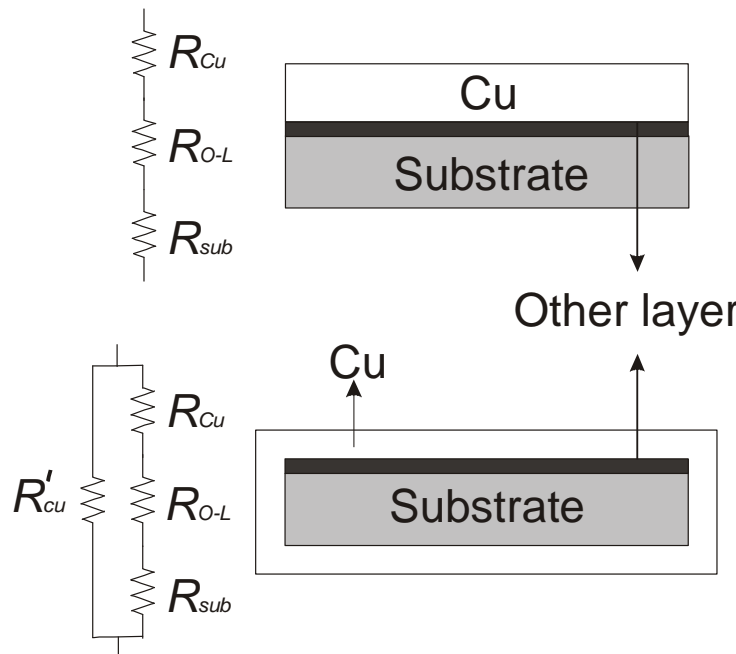

(b)

Fig. 3. Schematic drawing of stabilized CCs with associated equivalent thermal circuits. In (a), $\mathrm{Cu}$ is present only on one face of the tape and no parallel between thermal resistances is present. This is for example the case of the $\mathrm{CC}$ from Fujikura. In (b), $\mathrm{Cu}$ is present all around the tape, creating a parallel of thermal resistances. Case (b) particularly applies to $\mathrm{Cu}-$ electroplated tapes. 


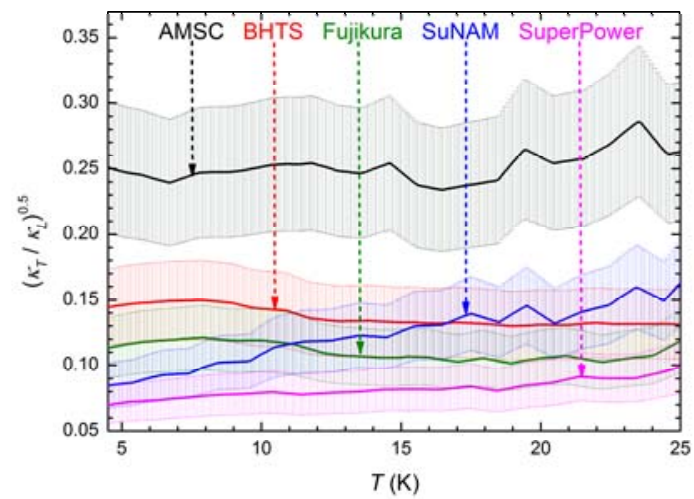

Fig. 4. Temperature dependence of the square root of the ratio $\kappa_{T} / \kappa_{L} . \kappa_{L}$ values of the investigated CCs have been extracted from [9]. Error bars are mostly determined by the uncertainty in $\kappa_{T}$ measurements.

passing through the lateral copper on varying the temperature.

We have shown here that the contribution from the lateral $\mathrm{Cu}$ to the overall $\kappa_{T}$ cannot be generally neglected in the case of electroplated $\mathrm{Cu}$. Nevertheless, a quantitative analysis of $\kappa_{T}$ is hindered by different factors. Fluctuations of the $\mathrm{Cu}$ thickness at the sample edges impede a precise determination of the dimensions of the lateral $\mathrm{Cu}$ strips. Furthermore, variation in morphology of $\mathrm{Cu}$ grains may arise from the deposition process and this has an influence on the local electron transport properties [19,20]. This confirms the importance of direct measurements in the determination of thermal properties of CCs.

We could not perform a quantitative analysis of $\kappa_{T}(T)$ for the $\mathrm{Cu}$-laminated $\mathrm{CC}$ from AMSC due to the lack of information on the material used to solder the two $\mathrm{Cu}$ strips on the edges.

From Fig. 2, we notice that the CC from AMSC has the highest $\kappa_{T}$ values. In this $\mathrm{CC}$, the substrate is made by NiW. The temperature dependence of the thermal conductivity of NiW has been reported in Ref. [21]. The better transverse thermal conduction properties of the $\mathrm{CC}$ from AMSC are correlated to the large amount of $\mathrm{Cu}$ used by this manufacturer (see Table II). In fact, the high thermal conductivity of $\mathrm{Cu}$ increases the overall $\kappa_{T}$.

The tape from BHTS exhibits the lowest $\kappa_{T}$. This is a consequence of $\mathrm{Cu}$ thickness and of the use of stainless steel (SS) as substrate material, being $\kappa_{S S}<\kappa_{\text {Hastelloy }}[11]$.

We have demonstrated that the contribution to thermal conduction of the $\mathrm{Cu}$ on the edges of the tape may affect the overall transverse thermal conduction. For the sake of completeness, we would like to point out that the fieldinduced reduction of $\kappa$ of $\mathrm{Cu}$ should also be taken into account, in case of use of the HTS in magnetic fields. Fieldinduced effects could considerably increase $R^{\prime} \mathrm{Cu}$, especially in case of high-RRR $\mathrm{Cu}[9,10]$.

In Fig. 4 we plot the square root of the ratio $\kappa_{T} / \kappa_{L}$, as calculated from the results of this work and $\kappa_{L}$ data reported in [9]. Without considering the contribution of the impregnation to the overall thermal transport in a magnet, these values can give valuable information on the anisotropy of the NZPV. Within the experimental uncertainty, most of the CCs have comparable values for $\left(\kappa_{T} / \kappa_{L}\right)^{0.5}$. Only the CC from AMSC shows larger values with respect to the other CCs. This result is related to the high $\kappa_{T}$ measured in this CC.

\section{CONCLUSION}

We have studied the transverse thermal conduction in REBCO coated conductors from different manufacturers. The use of an experimental setup specifically designed for thermal studies on technical superconductors has allowed us to measure $\kappa_{T}$ on single CCs and not on stacks of soldered tapes. This eliminates possible errors in the evaluation of $\kappa_{T}$ due to the influence of the solder. We have shown that the main contribution to the transverse thermal resistance comes from the substrate in the case of the $\mathrm{CC}$ from Fujikura, where $\mathrm{Cu}$ is present only on one side of the tape. For the CCs produced by the other manufacturers, $\mathrm{Cu}$ (solder for AMSC) on the tape edges opens parallel channels to thermal conduction that lower the overall thermal resistance. The comparison of transverse and longitudinal thermal conductivities of the CCs has allowed us to shed light on the anisotropy of the NZPV.

\section{ACKNOWLEDGMENT}

We acknowledge Christian Barth and Ciro Calzolaio for useful discussions, Damien Zurmuehle and Eugenie Gallay for technical support.

\section{REFERENCES}

[1] B. A. Albiss, I. M. Obaidat, "Applications of YBCO-coated conductors: a focus on the chemical solution deposition method," J. Mater. Chem. 20 (10), 2010, pp. 1836-1845.

[2] C. Senatore, M. Alessandrini, A. Lucarelli, R. Tediosi, D. Uglietti, Y. Iwasa Y, "Progresses and challenges in the development of high-field soleinodal magnets based on RE123 coated conductors," Supercond. Sci. Technol., 27, 2014, 103001 .

[3] N. T. Nguyen and P. Tixador, "A YBCO-coated conductor for a fault current limiter: architecture influences and optical study," Supercond. Sci. Technol., 23, 2010, 025008 .

[4] N. Bagrets, W. Goldacker, S. I. Schlachter, C. Barth, K.-P. Weiss, "Thermal properties of $2 \mathrm{G}$ coated conductor cable materials," Cryogenics 61, 2014, pp. 8-14.

[5] M. N. Wilson, Superconducting Magnets, Oxford: Clarendon, 1983.

[6] G. Manfreda, "Review of ROXIEs Material Properties Database for Quench Simulations," CERN Internal Note 2011-24 EDMS Nr:1178007, 2011.

[7] A. Devred, "General formulas for the adiabatic propagation velocity of the normal zone," IEEE Trans. Magnetics, vol. 25, no. 2, Mar. 1989, pp. 1698-1705.

[8] N. Bagrets, W. Goldacker, A. Jung, and K.-P. Weiss, "Thermal Properties of ReBCO Copper Stabilized Superconducting Tapes," IEEE Trans. Appl. Supercond., vol. 23, no. 3, Jun. 2013, Art. No. 6600303.

[9] M. Bonura, C. Senatore, "High-field thermal transport properties of REBCO coated conductors," Supercond. Sci. Technol., 2014, in press.

[10] J. G. Hust, A. B. Lankford, "Thermal conductivity of Aluminium, Copper, Iron, and Tungsten for Temperatures from $1 \mathrm{~K}$ to the Melting Point", National Bureau of Standards, Boulder, CO, NBSIR 84-3007, 1984.

[11] P. Bauer, H. Rajainmaki, E. Salpietro, "EFDA Material Data Compilation for Superconductor Simulation”, EFDA CSU, Garching, 04/18/07

[12] C. Lacroix, J.-H. Fournier-Lupien, K. McMeekin, and F. Sirois, "Normal Zone Propagation Velocity in 2G HTS Coated Conductor With High Interfacial Resistance," IEEE Trans. Appl. Supercond. vol. 23, no. 3, Jun. 2013, Art. No. 4701605. 
[13] C. Lacroix and F. Sirois, "Concept of a current flow diverter for accelerating the normal zone propagation velocity in $2 \mathrm{G}$ HTS coated conductors," Supercond. Sci. Technol., 27, 2014, 035003.

[14] A. Stenvall, R. Mikkonen, and Pavol Kovàč, "Relation Between Transverse and Longitudinal Normal Zone Propagation Velocities in Impregnated $\mathrm{MgB}_{2}$ Windings," IEEE Trans. Appl. Supercond. vol. 19, No. 2, pp. 2403-2406, 2009 .

[15] Y. G. Park, Y. J. Hwang, J. Y. Jang, H. J. Kim, W. S. Lee, H. C. Jo, H. M. Kim, Y. S. Yoon, and T. K. Ko, "Evaluation on Electrical and Thermal Characteristics of Multi-Stacked HTS Coated Conductor With Various Stabilizers," IEEE Trans. Appl. Supercond. vol. 24, no. 3, Jun. 2014, Art. No. 4700904

[16] M. Bonura, C. Senatore, "Thermal Conductivity of Industrial $\mathrm{Nb}_{3} \mathrm{Sn}$ Wires Fabricated by Various Techniques," IEEE Trans. Appl. Supercond. vol. 23, no. 3, Jun. 2013, Art. No. 6000404.

[17] Y. Iwasa, Case Studies in Superconducting Magnets, 2nd ed., Springer ISBN: 978-0-387-09799-2, 2009

[18] J. Lu, E. S. Choi, and H. D. Zhou, "Physical properties of Hastelloy ${ }^{\circledR}$ C$276^{\mathrm{TM}}$ at cryogenic temperatures," J. Appl. Phys. 103, 2008, 064908.

[19] H.D. Merchant, W.C. Liu, L.A. Giannuzzi, J.G. Morris, "Grain structure of thin electrodeposited and rolled copper foils," Materials Characterization 53, Issue 5, 2004, pp. 335-360.

[20] J. M. Camacho, A. I. Oliva, "Surface and grain boundary contributions in the electrical resistivity of metallic nanofilms," Thin Solid Films 515, 2006, pp. 1881-1885.

[21] N. Bagrets, M. Schwarz, C. Barth, K.-P. Weiss, "Thermal conductivity of materials used for preparation of the hybrid layered conductors based on high temperature superconductors," AIP Conference Proceedings 1435, 2012, pp. 281-285. 